\title{
A Series of Tetrathiafulvalene-Bismuth-Chlorides, Effects of Oxidation States of Cations on Structures and Electric Properties
}

Wen-Yu Yin, ${ }^{\dagger,+}$ Yi-Gang Weng, ${ }^{\dagger}$ Miao Jiang, ${ }^{\dagger}$ Shuai-Kang Yu, ${ }^{\dagger}$ Qin-Yu Zhu, ${ }^{* \dagger}$ and Jie Dai*广

${ }^{\dagger}$ College of Chemistry, Chemical Engineering and Materials Science, Soochow University, Suzhou 215123, P. R. China

Key Laboratory of Advanced Functional Materials; School of Chemistry \& Materials Engineering, Changshu Institute of Technology, Changshu, 215500, P.R. China

\section{Characterizations and measurements}

\section{Figures}

Figure S1. The experimental powder XRD patterns and the simulated pattern from the crystal data of compounds $\mathbf{1}-\mathbf{5}$.

Figure S2. The sketch map of the dihedral angle of the two five-membered rings of TMT-TTF molecule or cations.

Figure S3. Solid-state UV-vis absorption spectra of 1-5 in energy plot.

Figure S4. $1 / \mathrm{Cs}^{2}-\mathrm{V}$ plot for $\mathbf{1}, \mathbf{2}$, and $\mathbf{3}$ in dark and light.

Figure S5. Photocurrent density of the sample of 5 irradiated on and off with a $150 \mathrm{~W}$ xenon light under $0.5 \mathrm{~V}$ bias.

\section{Tables}

Table S1. Crystal data and structural refinement parameters for compounds 1-5.

Table S2. Short S $\cdots S, C \cdots S$ and $C \cdots C$ interaction in compounds 1-5.

Table S3. Slope constants for $\log J-\log V$ plot in Figure 5(c).

Table S4. The relative data to the calculation of the semiconductive properties for the samples of $\mathbf{1}, \mathbf{2}$, and $\mathbf{3}$. 


\section{Characterizations and measurements}

General Remarks. The preparation of TMT-TTF is prepared using the modified method reported in literature. ${ }^{1}$ All analytically pure reagents were purchased commercially and used without further purification. Elemental analyses $(\mathrm{C}, \mathrm{H}$ and $\mathrm{S})$ were performed using a VARIDEL III elemental analyzer. Solid-state room-temperature optical diffuse reflectance spectra of the microcrystal samples were obtained with a Shimadzu UV-2600 spectrophotometer using $\mathrm{BaSO}_{4}$ as a standard reference. Powder X-ray diffraction (PXRD) data were obtained using a D/MAX-3C X-ray diffraction meter with $\mathrm{CuK} \alpha(\lambda=1.5406 \AA)$ radiation. Electron spin resonance (ESR) spectra were carried out at room temperature and obtained using a Bruker ER-420 spectrometer with a $100 \mathrm{kHz}$ magnetic field in $\mathrm{X}$ band. Cyclic voltammetry (CV) experiments were performed on a CHI650 electrochemistry workstation in a three-electrode system with a Pt plate working electrode, a saturated calomel electrode (SCE) as reference electrode, and Pt wire as the auxiliary electrode.

X-ray Crystallographic Study. The crystal data were collected on a Bruker APEX-II CCD diffractometer for 1-5 and a Rigaku Mercury CCD diffractometer for 1' equipped with graphite monochromated Mo $\mathrm{K} \alpha(\lambda=0.71075 \AA)$ radiation were used to carry out the measurements at $200 \mathrm{~K}$. An absorption correction (multiscan) was applied for all these compounds. The structures were solved by direct methods using SHELXS-16 program and the refinements were performed against $F^{2}$ using SHELXL-16. ${ }^{2}$ Detailed crystal data and structural refinement parameters are listed in Table S1 in the Supporting Information.

\section{Device fabrication and measurements}

All the electrochemical measurements were performed on a CHI660E electrochemistry workstation in the ambient environment. The light source was a 150-W high pressure xenon lamp without filter, which was positioned $20 \mathrm{~cm}$ from the devices.

(a) Device and J-V measurements. 
A simple Schottky-type device was fabricated for all electrochemical measurements. The crystal samples were grinded into fine powder and a layer of the powder was paved an area of $0.500 \mathrm{~cm}^{2}$ on an ITO $(8 \Omega / \square)$ electrode with a mask. Another ITO electrode was covered on to be a sandwiched device and that was pressed by a clamp. The quantities of the samples were weighted as $1.5 \mathrm{mg}$ for all the compounds. The thickness of the sample layers were about $100 \mu \mathrm{m}$ measured by an electronic vernier caliper (Fowler). The $\mathrm{J}-\mathrm{V}$ plots were measured using the prepared devices in a two-electrode mode.

(b) Mott-Schottky plot measurements.

Mott-Schottky plots were obtained by impedance-frequency measurement with a three-electrode system. The working electrodes were prepared by a powder coating method. Weighed crystals of the compounds $(1.5 \mathrm{mg})$ were grinded and paved uniformly on an area of $0.500 \mathrm{~cm}^{2}$ ITO glass. A Pt plate and a saturated calomel electrode (SCE) were used as a counter electrode and a reference electrode. The supporting electrolyte solution was $0.1 \mathrm{~mol} \cdot \mathrm{L}^{-1}$ sodium sulfate aqueous solution. The capacitance of the semiconductor-electrolyte interface was collected at different frequency from 500 to $1000 \mathrm{~Hz}$.

(c) Calculation of the carrier density $(N)$ and the carrier mobility $(\quad)$.

The carrier density $N$ can be evaluated according to the Mott-Schottky equation (equation 2 ) by a linear plot of $1 / C^{2}-V$.

$$
\frac{1}{C^{2}}=\frac{2}{q \varepsilon_{r} \varepsilon_{0} N A^{2}}\left(V-V_{F B}+\frac{K T}{q}\right) \quad(\text { Equation } 1)^{3} \quad \text { (Mott-Schottky) }
$$

A linear Ohm J-V relation based on equation 1 is conducted:

$$
J=q N \mu_{0} \frac{V}{d} \quad(\text { Equation } 2)^{4}
$$

\section{References}

(1 ) Gemmell, C.; Janairo, G. C.; Kilburn, J. D.; Uecka, H.; Underhill, A. E. J. Chem. Soc. Perkin Trans., 1994, 2715-2720. 
(2) (a) Sheldrick, G. M. SHELXS-97, Program for structure solution; Universität of Göttingen, Göttingen, Germany, 1999. (b) Sheldrick, G. M. Crystal structure refinement with SHELXL. Acta Crystallogr., Sect. C: Struct. Chem. 2015, 71, 3-8.

(3) (a) Bera, B.; Chakraborty, A.; Kar, T.; Leuaa, P.; Neergat, M. Density of states, carrier concentration, and flat band potential derived from electrochemical impedance measurements of n-doped carbon and their influence on electrocatalysis of oxygen reduction reaction. J. Phys. Chem. C 2017, 121, 20850-20856. (b) Liu, C.; Wu, W.; Liu, K.; Li, M.; Hu, G.; Xu, H. Orientation growth and electrical property of CuSCN films associated with the surface states. CrystEngComm 2012, 14, 6750-6754.

(4) (a) Li, X.; Hove, M. V.; Zhao, M.; Bakeroot, B.; You, S.; Groeseneken, G.; Decoutere, S. Investigation on carrier transport through AlN nucleation layer from differently doped Si(111) substrates. IEEE T. Electron Dev. 2018, 65, 1721-1727. (b) Zhang, W.; Simoen, E.; Zhao, M.; Zhang, J. Analysis of leakage mechanisms in AIN nucleation layers on p-Si and p-SOI substrates. IEEE T. Electron Dev. 2019, 66, 1849-1855. 


\section{Figures}
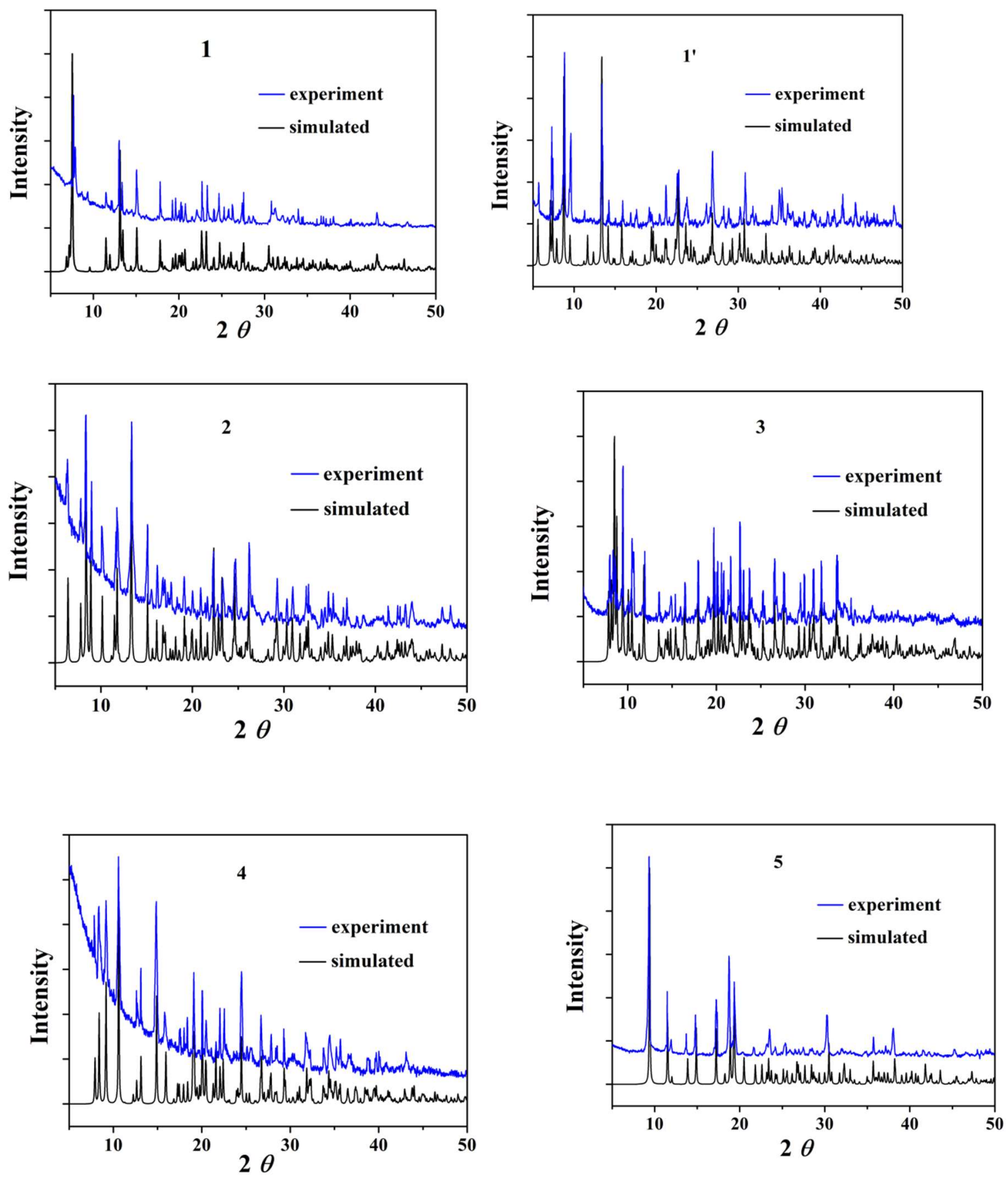

Figure S1. The experimental powder XRD patterns and the simulated pattern from the crystal data of compounds $\mathbf{1}-\mathbf{5}$. 


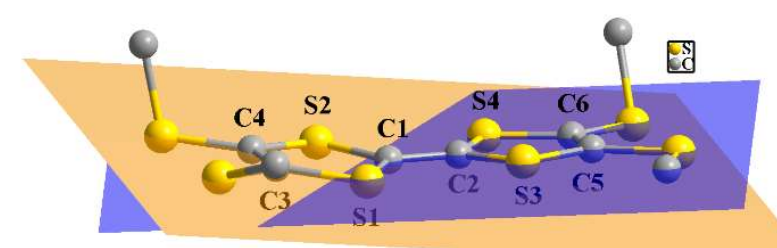

Figure S2. The dihedral angle of the two five-membered rings of TMT-TTF cation (plane 1: C1 C3 C4 S1 S2; plane 2: C2 C5 C6 S3 S4).

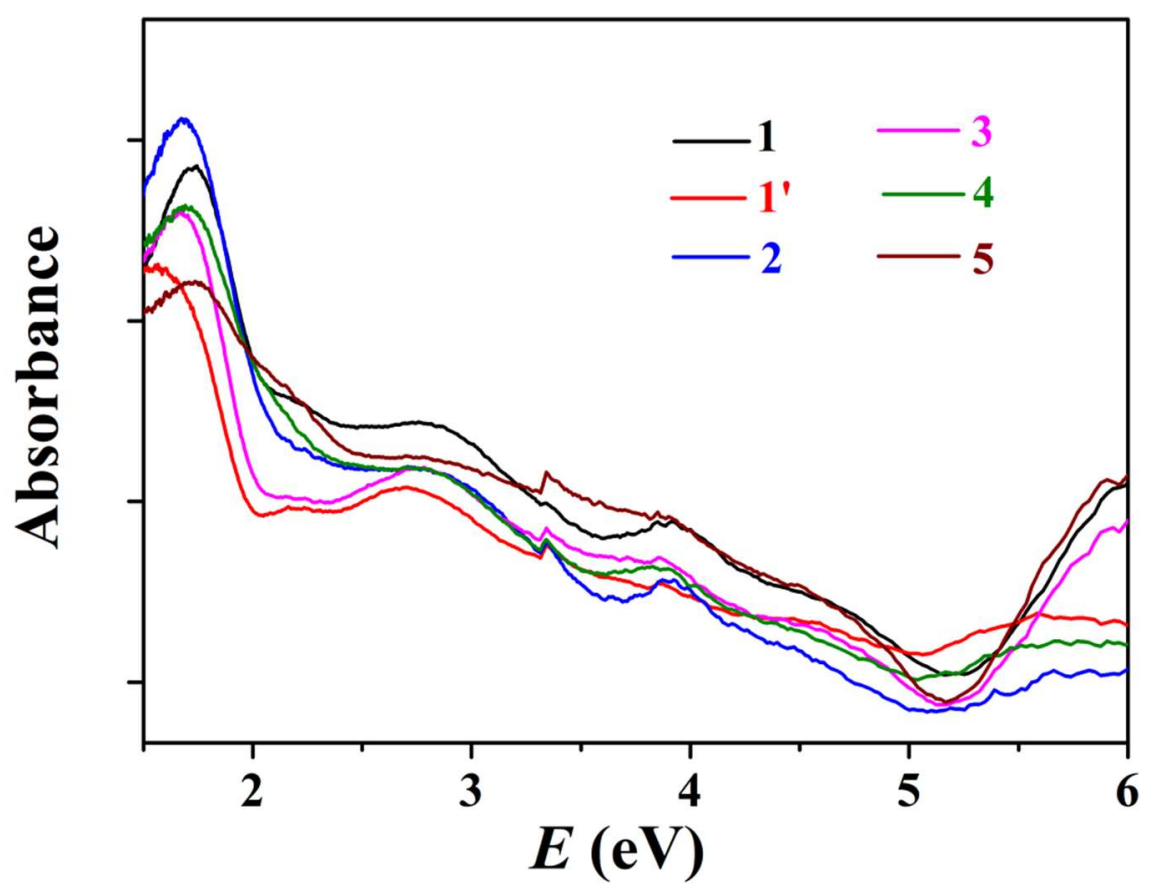

Figure S3. Solid-state UV-vis absorption spectra of 1-5 in energy plot. 

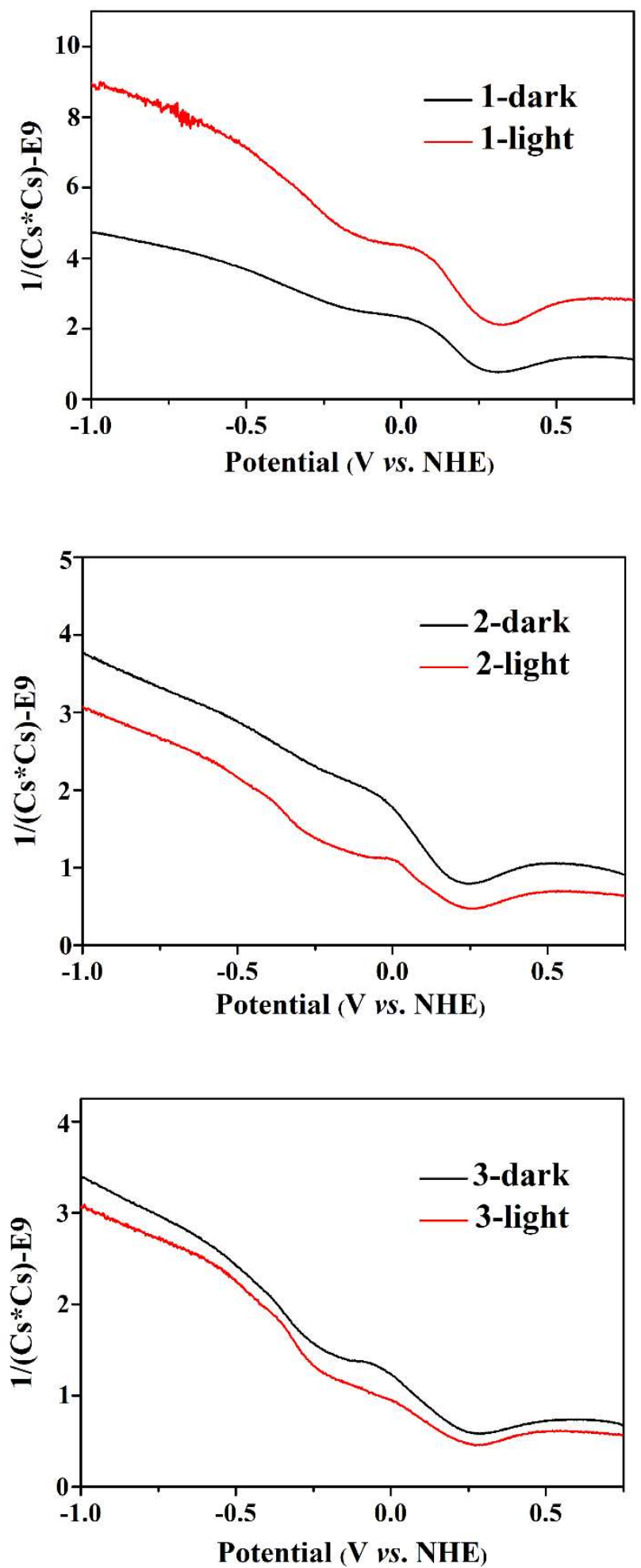

Figure S4. $1 / \mathrm{Cs}^{2}-\mathrm{V}$ plot for 1, 2, and 3 in dark and light. 


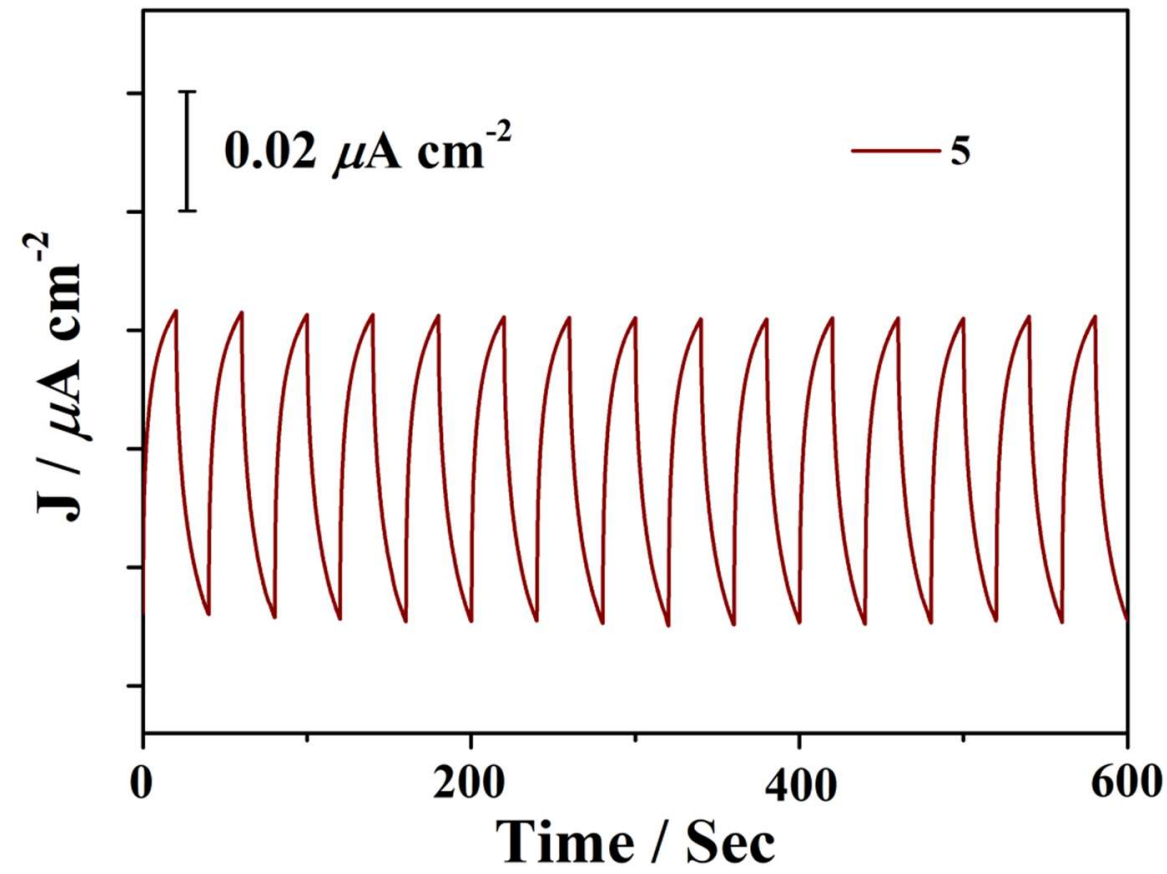

Figure S5. Photocurrent density of the sample of 5 irradiated on and off with a $150 \mathrm{~W}$ xenon light under $0.5 \mathrm{~V}$ bias. 
Table S1. Crystal data and structural refinement parameters for compounds 1-5.

\begin{tabular}{|c|c|c|c|}
\hline & 1 & 1 ' & 2 \\
\hline formula & $\mathrm{C}_{20} \mathrm{H}_{24} \mathrm{Bi}_{3} \mathrm{Cl}_{11} \mathrm{~S}_{16}$ & $\mathrm{C}_{20} \mathrm{H}_{24} \mathrm{Bi}_{3} \mathrm{Cl}_{11} \mathrm{~S}_{16}$ & $\mathrm{C}_{30} \mathrm{H}_{36} \mathrm{Bi}_{4} \mathrm{Cl}_{16} \mathrm{~S}_{24}$ \\
\hline fw & 1794.24 & 1794.24 & 2569.15 \\
\hline cryst size $\left(\mathrm{mm}^{3}\right)$ & $0.10 \times 0.10 \times 0.30$ & $0.20 \times 0.20 \times 0.40$ & $0.05 \times 0.07 \times 0.10$ \\
\hline cryst syst & triclinic & triclinic & triclinic \\
\hline space group & $P \overline{1}$ & $P \overline{1}$ & $P \overline{1}$ \\
\hline$a(\AA)$ & $14.442(3)$ & $11.952(2)$ & $11.1810(9)$ \\
\hline$b(\AA)$ & $14.648(3)$ & $13.851(3)$ & $12.0165(10)$ \\
\hline$c(\AA)$ & $14.755(3)$ & $17.478(4)$ & $15.3223(12)$ \\
\hline$\alpha(\mathrm{deg})$ & $108.171(6)$ & $112.78(3)$ & $70.7010(15)$ \\
\hline$\beta(\operatorname{deg})$ & $101.588(6)$ & $93.56(3)$ & $70.6230(15)$ \\
\hline$\gamma(\operatorname{deg})$ & $115.975(6)$ & $107.00(3)$ & $80.9250(16)$ \\
\hline$V\left(\AA^{3}\right)$ & 2449.1(9) & $2500.2(11)$ & $1830.2(3)$ \\
\hline Z & 2 & 2 & 1 \\
\hline$\rho_{\text {calcd }}\left(\mathrm{g} \mathrm{cm}^{-3}\right)$ & 2.433 & 2.383 & 2.331 \\
\hline$F(000)$ & 1672 & 1672 & 1204 \\
\hline$\mu\left(\mathrm{mm}^{-1}\right)$ & 11.558 & 11.808 & 10.883 \\
\hline$T(\mathrm{~K})$ & $200(2)$ & $200(2)$ & $200(2)$ \\
\hline reflns collected & 21863 & 18156 & 12580 \\
\hline unique reflns & 8552 & 11127 & 8293 \\
\hline observed reflns & 4328 & 7556 & 5674 \\
\hline no. params & 459 & 459 & 340 \\
\hline GOF on $F^{2}$ & 1.181 & 1.053 & 1.169 \\
\hline$R_{1}[\mathrm{I}>2 \sigma(I)]$ & 0.0555 & 0.0321 & 0.0560 \\
\hline${ }_{W} R_{2} \quad[\mathrm{I}>2 \sigma(I)]$ & 0.0983 & 0.0553 & 0.1539 \\
\hline
\end{tabular}




\begin{tabular}{|c|c|c|c|}
\hline & 3 & 4 & 5 \\
\hline formula & $\mathrm{C}_{20} \mathrm{H}_{24} \mathrm{Bi}_{3} \mathrm{Cl}_{13} \mathrm{~S}_{16}$ & $\mathrm{C}_{10} \mathrm{H}_{12} \mathrm{BiCl}_{5} \mathrm{~S}_{8}$ & $\mathrm{C}_{5} \mathrm{H}_{6} \mathrm{BiCl}_{4} \mathrm{~S}_{4}$ \\
\hline fw & 1865.27 & 774.91 & 545.12 \\
\hline cryst size $\left(\mathrm{mm}^{3}\right)$ & $0.10 \times 0.10 \times 0.20$ & $0.08 \times 0.10 \times 0.15$ & $0.05 \times 0.07 \times 0.10$ \\
\hline cryst syst & triclinic & triclinic & monoclinic \\
\hline space group & $P \overline{1}$ & $P \overline{1}$ & $I 2 / a$ \\
\hline$a(\AA)$ & 11.944(3) & $9.7401(12)$ & $7.7239(12)$ \\
\hline$b(\AA)$ & $12.893(3)$ & $10.6696(13)$ & $37.570(6))$ \\
\hline$c(\AA)$ & 19.971(5) & $11.4070(15)$ & $10.0050(13)$ \\
\hline$\alpha(\operatorname{deg})$ & $94.749(4)$ & $80.548(3)$ & 90 \\
\hline$\beta(\operatorname{deg})$ & $105.600(4)$ & $81.884(3)$ & $103.864(4)$ \\
\hline$\gamma(\operatorname{deg})$ & $116.106(4)$ & $86.871(2)$ & 90 \\
\hline$V\left(\AA^{3}\right)$ & $2587.1(10)$ & $1157.0(3)$ & $2818.7(7)$ \\
\hline Z & 2 & 2 & 8 \\
\hline$\rho_{\text {calcd }}\left(\mathrm{g} \mathrm{cm}^{-3}\right)$ & 2.394 & 2.224 & 2.569 \\
\hline$F(000)$ & 1740 & 736 & 2008 \\
\hline$\mu\left(\mathrm{mm}^{-1}\right)$ & 11.516 & 8.914 & 13.822 \\
\hline$T(\mathrm{~K})$ & $200(2)$ & $200(2)$ & $200(2)$ \\
\hline reflns collected & 21834 & 7677 & 8478 \\
\hline unique reflns & 15255 & 5117 & 2398 \\
\hline observed reflns & 10967 & 4356 & 1635 \\
\hline no. params & 477 & 221 & 141 \\
\hline GOF on $F^{2}$ & 1.064 & 1.032 & 1.167 \\
\hline$R_{1}[\mathrm{I}>2 \sigma(I)]$ & 0.0555 & 0.0376 & 0.0970 \\
\hline${ }_{W} R_{2} \quad[\mathrm{I}>2 \sigma(I)]$ & 0.1400 & 0.0902 & 0.2191 \\
\hline
\end{tabular}


Table S2. Short S $\cdots \mathrm{S}, \mathrm{C} \cdots \mathrm{S}$ and $\mathrm{C} \cdots \mathrm{C}$ interaction in compounds $\mathbf{1}-\mathbf{5}$.

\begin{tabular}{|c|c|c|c|c|c|c|c|c|}
\hline & $\mathrm{S} \cdots \mathrm{S}$ dis & nces $(\AA)$ & & & & & & \\
\hline \multirow[t]{2}{*}{1} & $\mathrm{~S} 1 \cdots \mathrm{S} 9$ & 3.369 & $\mathrm{~S} 2 \cdots \mathrm{S} 10$ & 3.276 & $\mathrm{~S} 3 \cdots \mathrm{S} 11$ & 3.579 & $\mathrm{~S} 4 \cdots \mathrm{S} 12$ & 3.388 \\
\hline & S5 $\cdots$ S13 & 3.483 & S6 $\cdots$ S14 & 3.382 & $\mathrm{~S} 8 \cdots \mathrm{S} 16$ & 3.509 & $\mathrm{~S} 9 \cdots \mathrm{S} 12$ & 3.581 \\
\hline $1 '$ & $\mathrm{~S} 5 \cdots \mathrm{S} 16$ & 3.331 & S8 $\cdots$ S 8 & 3.439 & & & & \\
\hline \multirow[t]{2}{*}{2} & $\mathrm{~S} 1 \cdots \mathrm{S} 8$ & 3.532 & $S 2 \cdots S 7$ & 3.471 & $\mathrm{~S} 3 \cdots \mathrm{S} 10$ & 3.473 & S4 $\cdots S 5$ & 3.425 \\
\hline & $\mathrm{S} 12 \cdots \mathrm{S} 12^{\prime}$ & 3.232 & & & & & & \\
\hline 3 & $\mathrm{~S} 6 \cdots \mathrm{S} 10$ & 3.512 & & & & & & \\
\hline 4 & - & & & & & & & \\
\hline 5 & - & & & & & & & \\
\hline
\end{tabular}

\begin{tabular}{|c|c|c|c|c|c|c|c|c|}
\hline \multicolumn{5}{|c|}{ C…S distances $(\AA)$} & \multicolumn{4}{|c|}{ C…C distances $(\AA)$} \\
\hline 1 & $\mathrm{C} 2 \cdots \mathrm{S} 12$ & 3.443 & $\mathrm{C} 6 \cdots \mathrm{S} 16$ & 3.478 & $\mathrm{C} 1 \cdots \mathrm{C} 11$ & 3.364 & $\mathrm{C} 1 \cdots \mathrm{C} 12$ & 3.231 \\
\hline & $\mathrm{C} 13 \cdots \mathrm{S} 5$ & 3.475 & $\mathrm{C} 4 \cdots \mathrm{S} 10$ & 3.436 & $\mathrm{C} 4 \cdots \mathrm{C} 14$ & 3.307 & $\mathrm{C} 3 \cdots \mathrm{C} 13$ & 3.363 \\
\hline 1 ' & - & & & & - & & & \\
\hline 2 & - & & & & - & & & \\
\hline 3 & - & & & & - & & & \\
\hline 4 & - & & & & - & & & \\
\hline 5 & - & & & & - & & & \\
\hline
\end{tabular}


Table S3. Slope constants for $\log \mathrm{J}-\log \mathrm{V}$ plot in Figure 5(c).

Equation $y=a+b * x$

Weight No Weighting

\begin{tabular}{llllll}
\hline \multirow{2}{*}{ Compound } & \multirow{2}{*}{$\begin{array}{l}\text { Residual Sum } \\
\text { of Squares }\end{array}$} & & Pearson's $\mathrm{A}$ & Adj. & \multicolumn{2}{c}{ Slope } \\
\cline { 5 - 6 } & & R-Square & Value & Standard Error \\
\hline $\mathbf{1}$ (in dark) & 0.76818 & 0.99968 & 0.99936 & 1.03105 & $3.37688 \mathrm{E}-4$ \\
$\mathbf{1}$ (in dark) & 1.19841 & 0.99949 & 0.99899 & 1.02703 & $4.2178 \mathrm{E}-4$ \\
$\mathbf{2}$ (in dark) & 1.21214 & 0.99947 & 0.99895 & 1.01271 & $4.24188 \mathrm{E}-4$ \\
$\mathbf{3}$ (in dark) & 5.23013 & 0.998 & 0.996 & 1.07755 & $8.81127 \mathrm{E}-4$ \\
$\mathbf{4}$ (in dark) & 2.13266 & 0.99908 & 0.99815 & 1.05276 & $5.8548 \mathrm{E}-4$ \\
$\mathbf{5}$ (in light) & 5.23013 & 0.998 & 0.996 & 1.07755 & $8.81127 \mathrm{E}-4$ \\
\hline
\end{tabular}

Table S4. The relative data to the calculation of the semiconductive properties for the samples of $\mathbf{1}, \mathbf{2}$, and $\mathbf{3}$.

\begin{tabular}{ccccccc}
\hline & $\mathbf{1}(\mathbf{D})$ & $\mathbf{1}(\mathbf{L})$ & $\mathbf{2}(\mathbf{D})$ & $\mathbf{2}(\mathbf{L})$ & $\mathbf{3}(\mathbf{D})$ & $\mathbf{3}(\mathbf{L})$ \\
\hline Cs & $2.076 \times 10^{-5}$ & $1.516 \times 10^{-5}$ & $2.369 \times 10^{-5}$ & $3.011 \times 10^{-5}$ & $2.845 \times 10^{-5}$ & $3.346 \times 10^{-5}$ \\
$k_{1}$ & $8.282 \times 10^{9}$ & $1.024 \times 10^{10}$ & $5.759 \times 10^{9}$ & $3.921 \times 10^{9}$ & $3.152 \times 10^{9}$ & $2.213 \times 10^{9}$ \\
$k_{2}$ & $8.40 \times 10^{-6}$ & $2.37 \times 10^{-5}$ & $1.09 \times 10^{-5}$ & $1.45 \times 10^{-5}$ & $3.15 \times 10^{-7}$ & $1.39 \times 10^{-6}$ \\
\hline
\end{tabular}

(D) in dark and (L) in light.

Units: $\mathrm{C}, \mathrm{F} \mathrm{cm}^{-1} ; k_{1}, \mathrm{~F} \mathrm{~cm} \mathrm{~V}^{-1} \mathrm{~s}^{-1}$. 